\title{
Public views on gene editing and its uses
}

To the Editor: Rapid advances in genome editing and its potential application in medicine and enhancement have been hotly debated by scientists and ethicists. Although it has been proposed that germline gene editing be discouraged for the time being ${ }^{1}$, the use of gene editing in somatic human cells in the clinical context remains controversial, particularly for interventions aimed at enhancement ${ }^{2}$. In a report on human genome editing, the US National Academies of Sciences, Engineering, and Medicine (NAS; Washington, DC) notes that "important questions raised with respect to genome editing include how to incorporate societal values into salient clinical and policy considerations" 3 . We report here our research that opens a window onto what the public thinks about these issues.

We conducted online quota sample surveys of more than 1,000 respondents in Austria, Denmark, Germany, Hungary, Iceland, Italy, the Netherlands, Portugal, Spain, UK (EEA-10 countries) and the United States ( $n=11,716$; Supplementary Note, section 1) to elicit judgments about gene editing using the contrastive vignette method $^{4,5}$. In our study, four vignettes in an experimental design combined two contexts and two recipient categories (Supplementary Note, section 2). The contexts were therapy (curing a disease) and enhancement (improving memory and learning capacity). The recipient categories were adult and prenatal. The vignettes presented brief accounts of situations leading to a decision to use gene editing. Each respondent read one of the four vignettes (adult therapy, prenatal therapy, adult enhancement or prenatal enhancement) assigned at random and was then asked, "Do you think he/they made a morally acceptable decision?" and "In his/their shoes would you make the same choice?" Responses were recorded on an 11point scale (from -5 for "No, definitely not" to +5 for "Yes, definitely"). Comparing the responses across vignettes reveals the effect of the experimental manipulations.
A multiple regression analysis (Table 1) shows that the therapy vignettes have, on average, higher scores than the enhancement vignettes on moral acceptability and on agreement that the respondent would make the same choice (to use gene editing) by over 4 points in the 11-point scale. The prenatal compared with the adult recipient elicits a lower assessment of moral acceptability, and lower agreement that the respondent would make the same choice. Adding age, gender and education level of the respondents to the regression showed only that female respondents were more cautious about gene editing in general.

Across the 11 countries in the study, support is consistently greater for treatment than enhancement (between 3.3 and 5.2 scale points). Similarly, there is greater support across all countries for intervention on adults than prenatals, but the magnitude effect of the target recipient is smaller (between 1.0 and 2.1 scale points). This is in agreement with the NAS report that there are "indications of public discomfort with using genome editing for what is deemed to be enhancement."

A notable feature of the responses to the vignettes is how the range of opinion varies across the targets and purposes of the intervention (Fig. 1). For both adult therapy and prenatal enhancement the responses show broad agreement. The former is accepted with a median response of 8 , whereas the latter is rejected with a median response just above zero. In contrast, adult enhancement and prenatal therapy appear to be morally ambiguous, reflected in very diverse opinions. $50 \%$ of the responses range over about half of the 11-point scale.

To explore the respondents' thinking we asked, "In a few words, can you tell us why you agree or disagree with the decision." Almost three out of four people added comments, suggesting that the topic is of importance. A systematic content analysis identified 21 broad themes (Supplementary Note, section 3).

For adult therapy, $75 \%$ of the comments were positive evaluations of gene editing technology. In order of frequency, these comments related to the following: it led to "improvements to quality of life"; it would enable "curing dementia"; and the "benefits outweighing the risks". For prenatal therapy the proportion of support for gene editing declines to $60 \%$. Positive comments for this type of therapy were the same as for 'adult therapy', but included additional comments, such as "it is natural for parents to want the best for their children." Gene editing for adult enhancement achieves only $26 \%$ positive comments. On the negative side, people mention there is "no need; being normal or average is OK," and that there might be "risks and unknown consequences." Only $11 \%$ of comments on prenatal enhancement are positive. In order of frequency, the negative remarks say gene editing is "unnatural and messing with nature"; that "there is no need" for this type of intervention; there are "risks of unknown consequences"; and it is just "wrong."

Might previous debates around modern biotech carry over into people's thinking about gene editing? For example, do

\begin{tabular}{|c|c|c|c|}
\hline Response variable & $\begin{array}{l}\text { Adult compared with } \\
\text { prenatal }\end{array}$ & $\begin{array}{l}\text { Enhancement compared } \\
\text { with therapy }\end{array}$ & $\begin{array}{l}\left(R^{2}\right) \text { Percentage of total vari- } \\
\text { ance in "Would you make the } \\
\text { same choice" accounted for by } \\
\text { the model }\end{array}$ \\
\hline $\begin{array}{l}\text { Is it morally accept- } \\
\text { able? }\end{array}$ & $1.89^{* *}$ & $-3.68 * *$ & 0.36 \\
\hline $\begin{array}{l}\text { Would you make the } \\
\text { same choice? }\end{array}$ & $1.47 * *$ & $-4.34 * *$ & 0.37 \\
\hline
\end{tabular}




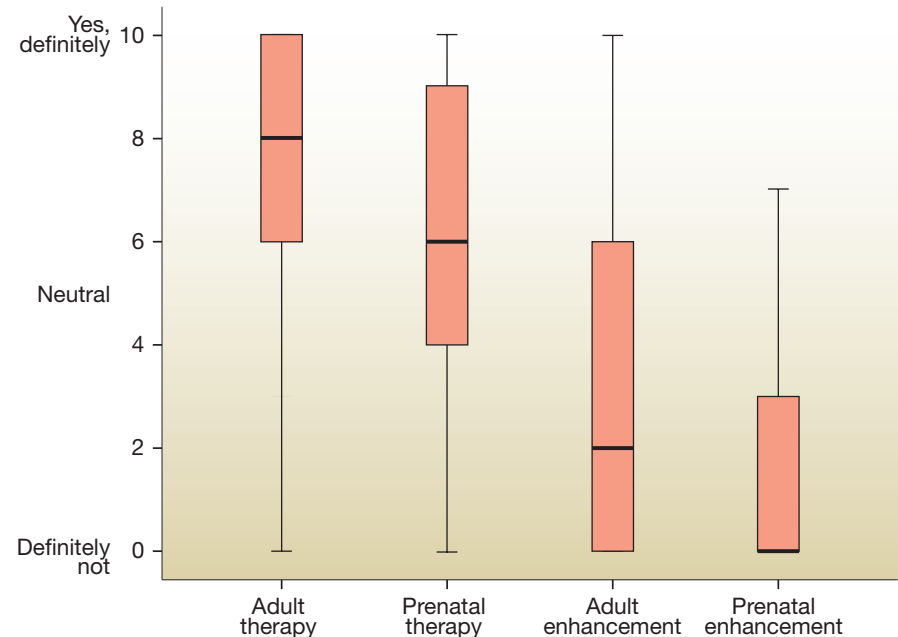

Figure 1 Would you make the same decision? Box plots of survey responses to each gene editing vignette (adult therapy, prenatal therapy, adult enhancement or prenatal enhancement). The boxes show the 25th to 75 th percentile responses and the median, the horizontal line $(n=11,716)$.

respondents view gene editing through a critical lens because of associations with genetically modified organisms (GMOs)? The answer is no; fewer than $3 \%$ mentioned GMOs. Other issues that did not feature beyond $1 \%$ or $2 \%$ included 'designer babies' and some of the ethical questions around human enhancement-increasing social disparities, obtaining an unfair advantage and undermining character.

Turning to differences between countries, we calculated the median scores for the four experimental vignettes (Fig. 2). We show the median rather than the mean, because in some countries at least half of the respondents gave a zero for the enhancement vignettes. The differing assessments of 'therapy' (between 5 and 9) versus 'enhancement' (between 0 and 4 ) highlight the fact that it is the application, rather than the technology itself, that is the critical issue for the public. Gene editing as applied to 'adult therapy' receives consistent support across all countries. And although there are differences between countries over the use of gene editing for prenatal therapy, it is supported in the majority of countries. More than half of the sample in Austria, Denmark, Germany, Hungary, Iceland, Italy, the Netherlands, Portugal and the UK say they would not use gene editing for prenatal enhancement. This pattern is also seen in Austria, Denmark and Germany for adult enhancement.

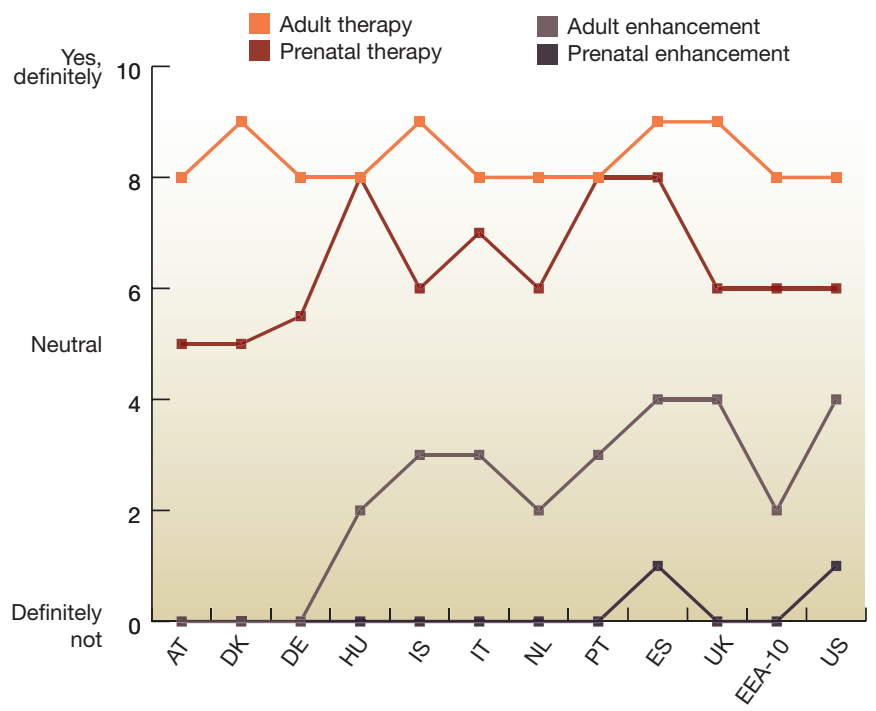

Figure 2 National differences on "Would you make the same decision?" ( $n=11,716)$. ES, Spain; PT, Portugal; NL, the Netherlands; IT, Italy; IS, Iceland; HU, Hungary; DE, Germany; DK, Denmark; AT, Austria.

Across countries, a stronger precautionary judgment is evident for gene editing for therapy and enhancement in the prenatal compared with the adult scenario. This is somewhat counterintuitive. For example, assuming successful use in the therapeutic context, gene editing at the prenatal stage will have greater positive impact on the future outcomes of the recipient, simply because the recipient will live fewer years with a medical disability, compared with intervention in adulthood. In the enhancement context, prenatal intervention will arguably cause less distress than in the adult context. In adults, concerns about the violation of the sense of self, or of personal authenticity, are often presented as reasons to avoid enhancement ${ }^{6,7}$. Concerns about authenticity violations in the prenatal context are likely to be less compelling to many people, although there may be other ethical considerations that again tip the balance. Such issues will need to be critically interrogated in the deliberation over the uses of gene-editing technology. Although the US public joins people in the UK and Spain in being a little less negative than other EEA-10 countries about adult enhancement, differences between the United States and the EEA-10 countries are notable by their absence.

As with many other technologies, the public's attention is on the applications or uses; these drive moral judgments. Yet scientific experts tend to focus on the technology itself. This harks back to the old struggle between regulating the process (the technology) or the applications (uses of the technology) that has caused so many problems for agricultural biotech in Europe ${ }^{8}$. Focusing on the technology will lead to inconsistent regulation, always lagging behind scientific progress. Focusing on uses will also present challenges: if countries opt for different regulations on the uses and target recipients of gene editing, some people may take to medical tourism. Should policy prioritize national interests or be transnational to reduce the risks associated with diverging policies? Perhaps it is time to set up a multinational institutional structure to guide innovative technological applications that are societally contentious.

A final word on the value of surveys in this controversial territory. Public opinion cannot and should not tell us what is right to do. However, as the NAS report notes, "Public participation should be incorporated into the policy-making process for human genome editing and should include ongoing monitoring of public attitudes, informational 
deficits, and emerging concerns about issues surrounding enhancement." This survey is a contribution to understanding the practical and contextual dimensions of the ethical question; how can gene-editing technology contribute to human flourishing?

Note: Any Supplementary Information and Source Data files are available in the online version of the paper.

Editor's note: This article has been peer-reviewed.

\section{ACKNOWLEDGMENTS}

This research was funded by the European

Commission as part of the study "Neuroenhancement, responsible research and innovation" Grant Agreement No: 321464 . The field work was conducted by Respondi. This study complied with the ethical regulations of the Research Ethics Committee of the London School of Economics. Data created during this research are openly available online at https:// zenodo.org/record/823634.

\section{COMPETING FINANCIAL INTERESTS}

The authors declare no competing financial interests.

George Gaskell ${ }^{1}$, Imre Bard ${ }^{1}$, Agnes Allansdottir ${ }^{2}$, Rui Vieira da Cunha ${ }^{3}$, Peter Eduard ${ }^{4}$, Juergen Hampel ${ }^{5}$, Elisabeth Hildt ${ }^{6}$, Christian Hofmaier ${ }^{5}$, Nicole Kronberger ${ }^{7}$, Sheena Laursen ${ }^{4}$, Anna Meijknecht ${ }^{8}$, Salvör Nordal ${ }^{9}$, Alexandre Quintanilha ${ }^{3}$, Gema Revuelta ${ }^{10}$, Núria Saladié ${ }^{10}$, Judit Sándor ${ }^{11}$, Júlio Borlido Santos $^{3}$, Simone Seyringer ${ }^{7}$, Ilina Singh ${ }^{12}$, Han Somsen ${ }^{8}$, Winnie Toonders ${ }^{13}$, Helge Torgersen ${ }^{14}$, Vincent Torre ${ }^{15}$, Márton Varju ${ }^{11}$ \& Hub Zwart ${ }^{13}$

${ }^{1}$ Department of Methodology, London School of Economics, London, UK. ${ }^{2}$ Toscana Life Sciences Foundation, Siena, Italy. ${ }^{3}$ Institute of
Molecular and Cellular Biology, Porto, Portugal. ${ }^{4}$ Experimentarium, Science Communication Centre, Copenhagen, Denmark. ${ }^{5}$ Center for Interdisciplinary Risk and Innovation Studies, Stuttgart University, Stuttgart, Germany. ${ }^{6}$ Center for the Study of Ethics in the Professions, Illinois Institute of Technology, Chicago, Illinois, USA. ${ }^{7}$ Department of Social and Economic Psychology Johannes Kepler University, Linz, Austria. ${ }^{8}$ Tilburg Institute for Law, Technology, and Society, Tilburg University, Tilburg, The Netherlands. ${ }^{9}$ Centre for Ethics University of Iceland, Reykjavik, Iceland. ${ }^{10}$ Centre on Science, Communication and Society Universitat Pompeu Fabra, Barcelona, Spain. ${ }^{11}$ The Center for Ethics and Law in Biomedicine Central European University, Budapest, Hungary.

${ }^{12}$ Department of Psychiatry and Oxford Uehiro

Centre University of Oxford, Oxford, UK.

${ }^{13}$ Institute for Science, Innovation and Society Radboud University of Nijmegen, Nijmegen, The Netherlands. ${ }^{14}$ Institute of Technology Assessment Austrian Academy of Sciences, Vienna, Austria. ${ }^{15}$ Centre for Neurobiology, International School for Advanced Studies, Trieste, Italy.

e-mail:g.gaskell@lse.ac.uk

1. Baltimore, D. et al. Science 348, 36-38 (2015).

2. Bosley, K.S. et al. Nat. Biotechnol. 33, 478-486 (2015).

3. US National Academies of Sciences, Engineering, and Medicine. Human Genome Editing: Science, Ethics and Governance (The National Academies Press, 2017).

4. Fitz, N.S. et al. Neuroethics 7, 173-188 (2014).

5. Hainmueller, J., Hangartner, D. \& Yamamoto, T. Proc. Natl. Acad. Sci. USA 112, 2395-2400 (2015).

6. Schermer, M. Bioethics 22, 355-363 (2008).

7. Parens, E. Hastings Cent. Rep. 35, 34-41 (2005).

8. Torgersen, H. et al. in Biotechnology: the Making of a Global Controversy, (eds. Bauer, M. \& Gaskell, G.) 21-94 (Cambridge University Press, Cambridge, UK, 2002).

\section{Query the merits of embryo editing for reproductive research now}

To the Editor: Recently, a team from the United States used CRISPR-Cas9 on viable human embryos to correct a gene mutation that causes hypertrophic cardiomyopathy, a heritable heart condition in which the ventricle walls thicken to hinder proper blood flow ${ }^{1}$. For many, this announcement brings closer to reality the prospect of editing disease-associated mutations in fertility clinic embryos intended for reproductive use. The study certainly raises many scientific uncertainties and questions. But we contend that it also brings to light some questionable value assumptions that have largely flown under the radar in the social discourse around embryo editing. Here, we call attention to some of these assumptions and suggest that additional human embryo editing research may not be adequately justified until these issues have at least been openly acknowledged and debated. To be clear, our discussion is meant to apply only to 'preclinical' embryo editing research: that is, to corrective nuclear genome editing research performed in vitro with an eye toward eventual reproductive use under favorable regulatory circumstances. Our points do not necessarily apply to human embryonic genome editing studies for fundamental research aimed at silencing genes to understand their function in early development.

\section{Gaps in current ethical standards}

Although the US team abided by the most recent ethical standards for human genome editing, there remain critical gaps in these standards. According to guidelines issued last year by the US National Academy of Sciences (NAS; Washington, DC) and the International Society for Stem Cell Research (ISSCR), germline editing of nuclear DNA is permissible only if scientific work is limited to in vitro embryo research; no edited human embryos ought to be implanted for reproduction at this time ${ }^{2,3}$. Unlike the ISSCR, however, the NAS goes further to specify ten necessary conditions under which, in the future, in vitro germline editing research might be permitted to cross over into first-in-human clinical trials for reproduction (see Table 1).

Among these requirements, we highlight the following three: (1) that there must be no reasonable alternatives to reproductive embryo editing; (2) that embryo editing be done only to prevent a serious disease or condition; and ( 9 in Table 1) that there be "continued reassessment of both health and societal benefits and risks, with broad ongoing participation and input by the public."

We believe these additional NAS conditions are significant not only because they allow for the very possibility of reproductive embryo editing in the future, but also because they offer guidance now for scientists pursuing in vitro embryo editing research aimed at possible future reproductive use. Conditions (1) and (2), for instance, should motivate researchers to deliberate carefully about which genetic diseases to target in their in vitro embryo editing studies. Researchers investigating the technical feasibility of reproductive embryo editing must first select a specific disease and then stick with it for the long haul. They cannot jump from one disease to another and still hope to have all the preclinical safety, efficacy, and proof-of-concept data necessary for the regulatory approval of any particular intervention. For example, if the US Food and Drug Administration (FDA; Rockville, MD) ever allows germline editing trials, it is likely to do so for a very specific guide RNA sequence on a CRISPR-Cas9 construct intended to correct a particular gene mutation. The FDA will not give researchers carte blanche to pursue just any generic approach to CRISPR-Cas9 germline editing in a clinical trials context. Therefore, disease selection and specialization at the very start of in vitro research is crucial for teams aspiring to develop any future embryo editing intervention. 